\title{
The Distribution of ESBL-Producing Enterobacteriaceae: Leicestershire UK Compared to Worldwide
}

\author{
Ruth Reid, Shivanthi Samarasinghe* and Atheena Varnakulasingam \\ De Montfort University, UK \\ *Corresponding author: Shivanthi Samarasinghe, Faculty of Health \& Life Sciences, De Montfort University, UK. \\ To Cite This Article: Shivanthi Samarasinghe. The Distribution of ESBL-Producing Enterobacteriaceae: Leicestershire UK Compared to \\ Worldwide. Am J Biomed Sci \& Res. 2019 - 3(1). AJBSR.MS.ID.000636. DOI: 10.34297/AJBSR.2019.03.000636
}

Received: May 11, 2019 | Published: May 21, 2019

\section{Introduction}

Beta-lactam antibiotics are the most frequently used antibiotic worldwide, therefore, bacterial resistance has continued to rise since their introduction due to a permanent selective force for resistance mechanisms [1,2]. Extended-spectrum $\beta$-lactamases (ESBLs) are mainly plasmid-encoded enzymes that are able to inactivate a variety of $\beta$-lactam antibiotics, including penicillins, 2nd-, 3rd- and 4th-generation cephalosporins and monobactams (e.g. aztreonam) [3]. However, ESBLs are generally susceptible to clavulanic acid (a beta-lactamase inhibitor), sulbactam and tazobactam [4]. There are two distinct classification systems for beta-lactamases: the Ambler molecular classification and the BushJacoby-Medeiros functional classification. The protein homology of the beta-lactamases is used to determine the Ambler scheme of four classes [4]. The four classes can then be differentiated further, classes A, C and D are serine beta-lactamases and class B are metallo-beta-lactamases. In contrast, the Bush-Jacoby-Medeiros functional scheme takes advantage of the functional properties of the beta-lactamase enzymes to classify them, for example, their substrate and inhibitor profiles [4]. The Ambler Scheme shall be used from here onwards.

Clinically, the most important class A ESBLs include: TEM, SHV and CTX-M [5]. The highest diversification and quickest evolution is within the CTX-M family of class A. The most important class D beta-lactamase is the OXA-type. The TEM, SHV and OXA type ESBL enzymes originate from point mutations within the plasmidmediated TEM-1, TEM-2, SHV-1 and OXA-10 enzymes. When first discovered in 1965, the TEM beta-lactamases substrate and inhibition profiles were comparable to SHV-1, for example, TEM1 is only capable of hydrolyzing penicillins and first generation cephalosporins. Since 1965, the TEM beta-lactamases have evolved and diverged, variants now have increased activity against extended spectrum cephalosporins, for example TEM-3 [4]. The SHV betalactamases have been suggested to have originated from Klebsiella spp. Like the TEM beta lactamases, the SHV family has evolved from resistance to broad-spectrum penicillins such as ampicillin, tigecycline and piperacillin but not to the oxyimino substituted cephalosporins to conferring an extended spectrum resistance today [4]. ESBLs of the TEM and SHV-type were most frequently found in the 80's and 90's and are mainly associated with hospital outbreaks of Klebsiella pneumoniae [6].

To this date, there have been over 172 different CXT-M enzymes discovered, classified into five groups: CTX-M-1, CTX-M-2, CTX-M-8, CTX-M-9, and CTX-M-25, based on their amino acid composition [5]. Being part of the class A ESBLS, it would be assumed that CTX-M producing enzymes would be related to TEM and SHV groups. However, this is not the case. Each of the CTX-M groups presents a 94\% amino acid homology within the group itself and $90 \%$ homology between the other CTX-M groups. The CTX-M family is unlike other ESBLs in that it includes a complex and non-homologous group of enzymes. It has been suggested by phylogenetic analysis that the CTX-M enzymes did not originate by mutations from plasmid mediated enzymes but through chromosomal mobilization of bla genes from the Kluyvera species through mobile genetic elements. Like the other ESBLs, after these genes had been incorporated, the CTX-M enzymes evolved through punctual mutations due to antibiotic selective pressure. This brought about the variants of CTX-Ms that can be seen today [5]. The OXA-type are ESBLs and are so named because of their oxacillin-hydrolyzing abilities [4].

OXAs are beta-lactamases that facilitate resistance to ampicillin, cephalothin, oxacillin and cloxacillin, however are resistant to the beta-lactamase inhibitor clavulanic acid and are generally encoded by plasmids. The first OXA ESBL is believed to have been discovered in Turkey, from P. aeruginosa isolates in a single hospital [4]. Here we will give a brief overview of the prevalence of ESBL-producing bacteria in the Leicestershire area of the UK and how the prevalence varies worldwide.

a) Epidemiology

b) Europe 
In the UK, the Surveillance Atlas of Infectious Diseases reports the level of E.coli resistant to third generation cephalosporins surveyed in 2016 to be $9.2 \%$. This is actually a decline in incidence since 2013. In comparison, the incidence level in Bulgaria in 2016 was $41.6 \%$. Other problematic areas in Europe include Cyprus (30.2\%), Italy (29.8\%) and Slovakia (29.7\%) [7]. In a recent study in Leicestershire UK, we found that $71.6 \%$ of ESBL-producing urinary tract infection isolates were of the CTX-M type, with TEM, SHV and OXA detected in $\leq 16 \%$ of isolates. Multiple ESBLs were also found in a number of isolates. Looking in to the CTX-M group further, we found that CTX-M-1 was present in $84.1 \%$ of isolates. We also found that CTX-M-producing isolates had a significant correlation with the plasmids IncL/M, IncFII, IncFIA, IncN. Again multiple plasmids were common [8-14]. This is in correlation with what is found in the rest of the UK. Concerning the rest of Europe, different CTX-M subtypes are common in different countries. CTX-M-9 is common in Spain, whilst CTX-M-3 is common in Eastern Europe. Overall in Europe, the CTX-M-1 group (including CTX-M-15) is the most widespread and has been associated with isolates found in the community [4].

\section{United States of America}

It was estimated in 2001 that $5.3 \%$ of E.coli in America was ESBL-producing. A more recent study in 2009 in a cancer center revealed $9 \%$ of E.coli isolates were ESBL-producers.

\section{Asia and the Indian Subcontinent}

It has only been recently that we have begun to understand the degree to which ESBL-producing Enterobacteriaceae is effecting parts of Asia and the Indian subcontinent, and the incidence of ESBLs is continuing to rise [4]. In India specifically, three medical centers reported that $66 \%$ of third generation cephalosporin resistant Enterobacteriaceae harbored CTX-M-15. Ten other centers in India reported ESBL rates of 70\% within Enterobacteriaceae [4].

\section{The Middle Eastern Countries}

Incidences of $26 \%$ for ESBL-producing Klebsiella pneumoniae isolates have been seen in Saudi Arabia, whereas a prevalence of $26.5 \%$ for E. coli and 43\% for Klebsiella has been reported in Iran [15]. A different study in Iran reported that $42.1 \%$ of the E. coli isolates derived from patients with clinical symptoms of urinary tract infections were ESBL-producing [16].

\section{Africa}

In Tanzania, hospitals have described rates of ESBL-producing isolates in Klebsiella pneumoniae as high as $64 \%$. An orphanage in Mali reported that $100 \%$ of the children carried ESBL-producing Enterobacteriaceae [4]. Likewise, in Madagascar, it was observed that $10 \%$ of non-hospitalised patients carried ESBLs. It has been suggested that poverty is an important risk factor for carriage of ESBLs [4]. A systematic search review that used Twenty-six studies (409215 isolates) from 13 African countries ESBL-producing isolates from a urinary source varied from $1.5 \%$ to $22.8 \%$ [17]. Combined, these reports may indicate that the prevalence in Africa may be increasing.

\section{Conclusion}

As can be seen here, there is substantial global variation in the incidence of antibiotic resistance. This variation has been linked to the usage of antibiotics. Some countries have been working hard to reduce their antibiotic consumption, whereas others have seen a rapid rise in usage. Despite the variation in antibiotic consumption and resistance rates, it is clear that antibiotic resistance is a concern worldwide.

\section{Acknowledgement}

This work was supported by the De Montfort University PhD scholarship for Reid R. and MSc by Research Scholarship fund for Varnakulasingam A.

\section{References}

1. Pitout J, Sanders C, Sanders W (1997) Antimicrobial resistance with focus on beta-lactam resistance in gram-negative bacilli. Am J Med 103(1): 51-59.

2. Bush $K$, Macielag $M(2010)$ New $\beta$-lactam antibiotics and $\beta$-lactamase inhibitors. Expert Opin Ther Pat 20(10): 1277-1293.

3. Zurfluh K (2015) Replicon typing of plasmids carrying blaCTX-M-15 among Enterobacteriaceae isolated at the environment, livestock and human interface. Sci Total Environ 521-522: 75-78.

4. Shaikh S (2015) Antibiotic resistance and extended spectrum betalactamases: types, epidemiology and treatment. Saudi J Biol Sci 22(1): 90-101.

5. Rahman U (2018) The Growing Genetic and Functional Diversity of Extended Spectrum Beta-Lactamases. BioMed Research International 9519718.

6. Canton R (2012) CTX-M enzymes: origin and diffusion. Front Microbiol $3(110)$.

7. European Centre for Disease Prevention and Control (2017). Surveillance Atlas of Infectious Diseases.

8. Reid R, Al-Bayati M, Samarasinghe S (2018) Genotypic Identification of Extended-Spectrum $\beta$-Lactamase (ESBL) Producing Enterobacteriaceae from Urinary Tract Infections in the Leicestershire Area, United Kingdom: A One Health Prospective. Journal of Infectious Diseases and Diagnosis 3(2): 1000122.

9. Bonnet $R$ (2004) Growing group of extended-spectrum beta-lactamases: the CTX-M enzymes. Antimicrob Agents Chemother 48(1): 1-14.

10. Decousser J, Poirel L, Nordmann P (2001) Characterization of a chromosomally encoded extended-spectrum class A beta-lactamase from Kluyvera cryocrescens. Antimicrob Agents Chemother 45(12): 3595-3598.

11. Humeniuk C, Arlet G, Gautier V, Grimont P, Labia R, et al. (2002) Betalactamases of Kluyvera ascorbata, probable progenitors of some plasmid-encoded CTX-M types. Antimicrob Agents Chemother 46(9): 30145-3049.

12. Naas T, Oxacelay C, Nordmann P (2007) Identification of CTX-M-type extended-spectrum-beta-lactamase genes using real-time PCR and pyrosequencing. Antimicrob Agents Chemother 51(1): 223-230.

13. Peirano GPJ (2010) Molecular epidemiology of Escherichia coli producing CTX-M $\beta$-lactamases: the worldwide emergence of clone ST131 025:H4. Int J Antimicrob Agents 35(4): 316-321.

14. Shin J, KO KS (2015) Effect of plasmids harbouring blaCTX-M on the virulence and fitness of Escherichia coli ST131 isolates. Int J Antimicrob Agents 46(2): 214-218.

15. Tawfik A, Alswailem A, Shibl A, Al-Agamy M (2011) Prevalence and genetic characteristics of TEM, SHV, and CTX-M in clinical Klebsiella 
pneumoniae isolates from Saudi Arabia. Microb Drug Resist 17(3): 383388.

16. Ebrahim-Saraie HS, Nezhad NZ, Heidari H, Motamedifar A, Motamedifar M (2018) Detection of Antimicrobial Susceptibility and Integrons Among Extended-spectrum $\beta$-lactamase Producing Uropathogenic Escherichia coli Isolates in Southwestern Iran. Oman Med J 33(3): 218-223.
17. Tansarli G, Poulikakos P, Kapaskelis A, Falagas M (2014) Proportion of extended-spectrum $\beta$-lactamase (ESBL)-producing isolates among Enterobacteriaceae in Africa: evaluation of the evidence--systematic review. J Antimicrob Chemother 69(5): 1177-1184. 